\title{
Associative priming in faces: Semantic relatedness or simple co-occurrence?
}

\author{
MATEI VLADEANU, MICHAEL LEWIS, and HADYN ELLIS \\ Cardiff University, Cardiff, Wales
}

\begin{abstract}
In two experiments, we explored the effects of co-occurrence and semantic relationships in the associative priming of faces. In Experiment 1, pairs of computer-generated human faces were presented simultaneously (i.e., they co-occurred) with no associated semantic information attached to them. A significant facilitation effect in the subsequent recognition of these paired faces (priming) was observed. Thus, repeatedly presenting faces together while keeping semantic information to a minimum appears to be enough to produce associative priming. In Experiment 2, the computer-generated faces were associated with semantic information and again presented in pairs. Priming effects arising from co-occurrence and semantic relatedness were observed. The results from these experiments show that semantic relatedness is not the sole cause of the association between faces; co-occurrence plays a crucial role too. This conclusion has significant implications for the current computational models of face processing.
\end{abstract}

Contemporary views of associative face priming have been much influenced by the idea that a certain amount of semantic information must be shared between two associated faces in order to produce a facilitatory recognition effect. Since the first theoretical approaches to face priming (e.g., Bruce \& Valentine, 1986; Hay \& Young, 1982), parallels have been drawn to similar phenomena reported in the word processing literature. Priming occurs for semantically related words (e.g., bread and butter, knife and fork), it is suggested, because these words have much semantic information in common. According to Collins and Loftus's (1975) semantic network/spreading activation model, familiar concepts are represented as nodes in a semantic network. The node DOG, for example, is linked to other nodes that help define its meaning, such as TAIL, FUR, and MAMMAL. These nodes, however, are also linked to the word CAT. the fact that DOG and CAT prime each other is explained by the fact that they share semantic nodes. Bruce and Valentine, employing the same logic, argued that the face of Prince Charles, for example, facilitates the recognition of Princess Diana's face because they were linked by a number of common semantic features (e.g., they were British, part of the British royal family, and famous).

Although repeated co-occurrence is another possible explanation of the priming effect, Bruce and Valentine (1986) did not explore this suggestion further. Instead,

Correspondence concerning this article should be addressed to M. Lewis, Cardiff University, School of Psychology, Cardiff CF10 3YG, Wales (e-mail: lewismb@cardiff.ac.uk).

Note-This article was accepted by the previous editorial team, when Colin M. MacLeod was Editor. they proposed that the magnitude of the priming effect in known faces is due to the associative strength between the semantic representations of the faces. Their mere co-occurrence in the attentional field of the public was thought to have an influence, but not to be the cause of association. Bruce and Valentine's view became the accepted view in the face processing literature, so that associative priming was referred to as semantic priming and shared semantic information was thought to be the sole cause of association. In almost all published papers in the face priming literature, the same logic was employed to explain their results, thus strengthening the idea that associative priming is semantic in nature. In the present article, we argue that semantic and associative relationships are essentially different. In fact, the two forms of priming are often confounded in the face priming literature. Here, we refer to associative face priming as the form of priming produced by the repeated co-occurrence of stimuli, and to semantic face priming as the form of priming based on a purely semantic relation between the stimuli.

In the word literature, the distinction between associative and semantic priming has been acknowledged and has generated numerous debates. Lupker (1984) argued that "a purely semantic relationship between prime and target can provide very little automatic facilitation in a naming task" (p. 720). Lupker also proposed that semantic relatedness may augment the amount of priming obtained from an associative relationship. Thus, the author proposed that "associative relationships" are the main cause of what is generally referred to as semantic priming. More recently, Alario, Segui, and Ferrand (2000) arrived at a similar conclusion after conducting two studies of word-picture priming. In contrast, Lucas (2000), using a meta-analytic technique, summarized a considerable number of studies of semantic priming and concluded that a semantic prim- 
ing effect can occur in the absence of association, whereas associative priming cannot be obtained in the absence of a semantic relationship. However, Hutchison (2003) challenged this conclusion, arguing that it is difficult to distinguish between associative and semantic priming effects because highly associated words tend to share semantic relations as well.

In the face priming literature, the issue of associative versus semantic priming has not been fully investigated. As has been stated above, the general view in the face priming literature is that the priming effect can be explained solely by semantic relatedness. This view was heavily influenced by the development and publication of the computational models of face recognition. One of the most influential computational models of face recognition, the interactive activation and competition (IAC) model (Burton, Bruce, \& Johnston, 1990), was proposed as a theoretical framework for the previously published experimental work in face recognition, including associative priming. As is depicted in Figure 1, the IAC model contains units arranged in pools and linked by fixed-weight bidirectional links. The first pool contains the face recognition units (FRUs), which are activated by visual input and respond specifically to faces. For each face one knows, there is one FRU. Similar pools of units, not depicted in Figure 1, exist for other modalities (e.g., voice recognition units for auditory input). The second layer contains the person identity nodes (PINs), which form a multimodal gateway connecting different modalities (visual or auditory) to the semantic information units (SIUs). The SIUs comprise the third layer and store semantic information. Thus, the PINs constitute the first stage of person recognition, the decision about the familiarity of a face being made at the PIN level. Each FRU is connected to one associated PIN. PINs, however, can be connected to one or more SIUs (for a detailed description of the IAC and its functionality, see Burton et al., 1990).

Burton et al.'s (1990) IAC model assumes that two PINs could share one or more SIUs. Thus, the only way in which faces could be associated is by semantic mediation. Although Bruce and Valentine (1986) acknowledged the possibility that associative links could be formed through co-occurrence, the IAC model does not include any mechanism to allow this. Due to its architecture, the IAC cannot explain any association that is not semantically mediated.

Is the idea of semantic priming a mere IAC artifact, or is it experimentally derived? Bruce and Valentine (1986) noticed that the priming effect obtained in their experiment was significantly larger (100 msec or more) than the semantic priming effects reported in the word recognition literature (about $50 \mathrm{msec}$ on average). Other researchers reported larger priming effects in object recognition (e.g., Kroll \& Potter, 1984), but this effect is probably due to an increased similarity in the visual appearance and feature overlap of the objects. This explanation cannot be applied to Bruce and Valentine's experiment to explain the priming effect and its size because the faces they used (e.g., those of Prince Charles and Princess Diana) were quite

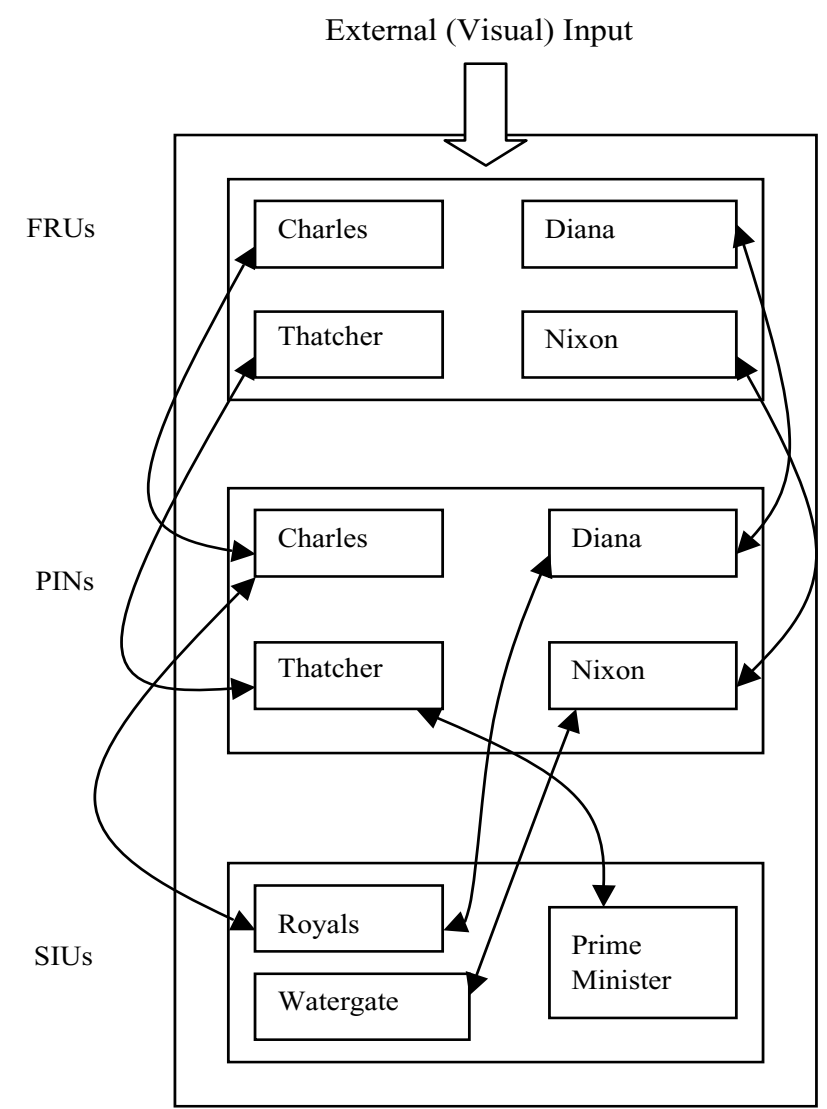

Figure 1. A simplified example of an IAC network. FRUs, face recognition units; PINs, person identity nodes; SIUs, semantic information units.

dissimilar with regard to visual appearance. Thus, since a perceptual explanation of the priming effect cannot be employed, Bruce and Valentine concluded that the data represented further evidence that the semantically based explanation must be valid. This conclusion is conveniently accommodated by the IAC model. Since the FRUs are considered to represent the perceptual level of the model, and since priming is hypothesized to take place at the PIN level, their conclusion was interpreted as support for the model and for how it simulates associative priming (i.e., through the SIUs).

In several other experiments, the possibility that associative priming is based on a perceptual mechanism was investigated and rejected. For example, a biologically based approach to face priming comes from Schweinberger's $(1995,1996)$ studies, in which within-domain (faceface) and cross-domain (name-face) associative priming was investigated by recording both response times and event-related brain potentials (ERPs). The ERP modulations due to priming for faces and those due to priming for names were found to be topographically equivalent, so Schweinberger concluded that associative priming effects are independent of the nature of the primes (names or faces). This automatically led to the conclusion that the locus of priming is not at a perceptual level. In addition 
to supporting claims from previous research that priming effects are due solely to associative relatedness and do not arise because of perceptual similarities, Schweinberger's research is also consistent with Burton et al.'s (1990) IAC model and the hypothesis that priming occurs at the PIN level. Since the IAC architecture hypothesizes that the PINs can be accessed through the SIUs, Schweinberger's studies also provide support for the idea that associative priming is semantic in nature.

Although widely accepted, the model cannot accommodate some recent findings. For example, Young, Flude, Hellawell, and Ellis (1994) tried to determine whether mere membership in a certain category (e.g., both target and prime faces are of actors, but the actors have never been seen in the same movie) is enough to produce priming or whether, instead, prime-target associative strength is the essential factor (e.g., in their study, Mel Smith and Griff Rhys-Jones - a popular British comic duo at the time - are strong associates). They discovered that inclusion within the same category is not enough to produce priming, whereas associative relatedness is a strong predictor of priming. Although this study clearly pointed out a major flaw in the design of the IAC model, subsequent studies continued.

Barry, Johnston, and Scanlan (1998) reported a reliable priming effect based on association but not on semantic category (e.g., members of a well-known British comedy duo would prime each other, but unrelated comedians would not). However, rather than focus on the fact that members of a comedy duo would co-occur, Barry et al. proposed that the semantic representations of people are collections of detailed biographical data, which the authors called BIOG units. These biographical units become associated through common episodic events (e.g., Michael Douglas is married to Catherine Zeta-Jones; Oliver Hardy and Stan Laurel always appeared together in the same movies). As the authors put it, "the interrelationships of representations of people are structured more along what could be called 'social' rather than abstract 'semantic' networks” (Barry et al., 1998, p. 877). Nevertheless, in our opinion BIOG units are a type of SIUs, since they essentially store meaningful (i.e., semantic) information. This brings a new issue into discussion - namely, what constitutes semantic information? Although it is not the aim of this article to investigate this issue, we would argue that if semantic memory is thought to comprise our structured world knowledge, then BIOG units are definitely part of semantic memory.

Like Bruce and Valentine (1986), Barry et al. (1998) acknowledged co-occurrence as a possible cause of association, but they integrated it into a distinct episode of memory. As we argued before, it is not entirely clear what information about a person qualifies for storage within the BIOG units and what is semantically abstract. To a certain extent, what is known about a person relates to appearance, biographical facts, and episodic memories. Cooccurrence is more likely to be registered automatically than to be recorded explicitly. Sometimes co-occurrence can also be derived from other biographical facts. For example, partnership could be regarded as biographical information, but it also implies a high degree of cooccurrence. This raises the possibility that co-occurrence is determined essentially by a functional relation. In other words, some people co-occur for a reason (e.g., they are partners), and this semantic information is used to associate them with each other. If this is true, then associative priming is indeed semantically mediated. This idea has been investigated in the word priming literature (see, e.g., Moss, Ostrin, Tyler, \& Marslen-Wilson, 1995). Moss et al. proposed that some words (specifically, words pertaining to instruments and scripts) that were traditionally thought to be related associatively in fact have a functional relationship. For example, hammer and nail do co-occur often, but they do so because the function of the hammer is to perform an action on the nail. Because it is very difficult to isolate the individual influence of the functional relationship from the influence of co-occurrence, in the present experiments we attempted to minimize the effect of potential functional relationships by using unknown faces that co-occur. If the faces are of unknown people, then no previous knowledge (including, e.g., biographical information about partnership) is available and, thus, a purely associative relation induced by repeated co-occurrence can be formed.

In contrast to Barry et al. (1998), Carson and Burton (2001) reported a priming effect based solely on category membership. Carson and Burton considered two people to be related (1) associatively if they often co-occurred, (2) categorially if they "share any particular personal information," and (3) semantically if "either, or both, of the previous relations are true. So this relation does not distinguish between categorial and associative relations" (Carson \& Burton, 2001, p. 1158). Since the authors acknowledged co-occurrence as a possible cause of association, it is not very straightforward why they chose to label two associated people (i.e., people that often co-occur) as "semantically related." As in the previous studies briefly described in the present work, the terms associative and semantic seem to be confounded, and, although Carson and Burton explicitly acknowledged the possible influence of co-occurrence, they still included it under the term semantic relatedness.

The central question of this article concerns how associations are formed. All the face priming studies briefly presented here can be used to argue in favor of the fact that semantic information is critical to associative priming. Regardless of how it is explained, the idea remains that a certain amount of semantic information has to be shared between two faces in order to produce an associative priming effect. The IAC model, which has dominated theoretical approaches to face processing for over a decade, is based on the semantic relatedness assumption as well. Although the role of co-occurrence has been acknowledged in the literature as a possible cause of association (see, e.g., Bruce \& Valentine, 1986; McNeill \& Burton, 2002), no significant effect has been reported on the basis of co-occurrence exclusively. An important point to bear in mind, however, is that in all the studies cited 
here faces and names of celebrities were used as stimuli. It could be argued that these faces are indeed semantically related but also share a variable degree of co-occurrence. Thus, we would argue that the degree of association between two people cannot be based on semantic relatedness alone. In this article, our goal is to explore two main theoretical issues. First, we will argue that semantic and associative priming are essentially two different forms of priming that are often confounded in the face priming literature. Semantic priming is based on semantic relationships, whereas associative priming is based mainly on co-occurrence. We will test this hypothesis by attempting to produce priming based on co-occurrence while keeping semantic relatedness to a minimum and, vice versa, to produce automatic priming based on semantic similarity while keeping co-occurrence to a minimum. Second, we hypothesize that in most studies conducted with known people (celebrities) both forms of priming contribute to the overall effect reported, but they overlap because strong associates share both semantic information and a high degree of co-occurrence. We predict that priming based on both semantic relatedness and co-occurrence will be significantly larger than that based on either of the two alone.

The first experiment was designed to investigate whether or not an associative priming effect can be obtained via co-occurrence alone, without any influence from semantic information. The second experiment was designed to investigate whether or not a similar facilitatory effect (i.e., priming) can be obtained without cooccurrence, on the basis only of semantic relatedness, and whether co-occurrence and semantic relatedness interact.

\section{EXPERIMENT 1}

In this experiment, computer-generated artificial faces were used as stimuli. Because the artificial faces did not have any semantic background, the role of semantic relatedness between the faces was kept to a minimum and, therefore, the role of co-occurrence could be manipulated and thereby fully explored.
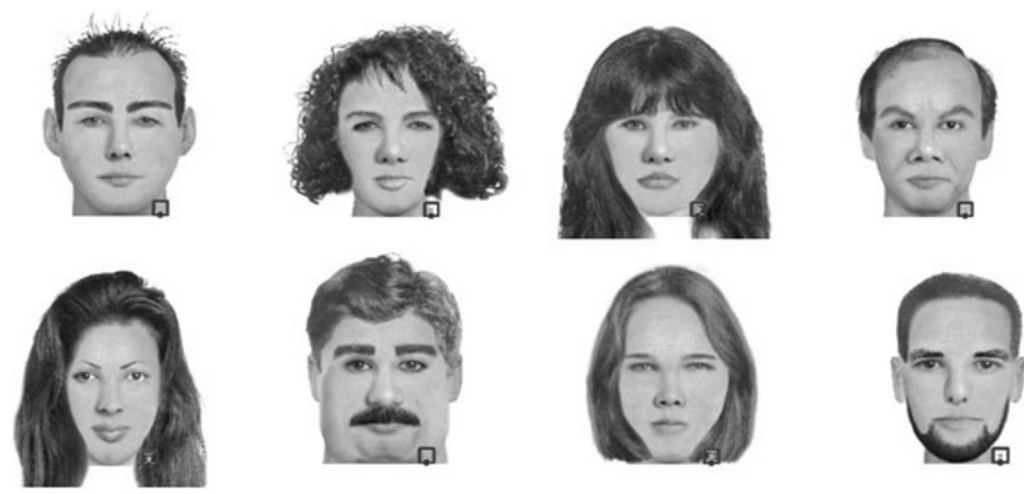

Figure 2. Examples of faces used in Experiments 1 and 2, designed using the InterQuest software package.

\section{Method}

Participants. Thirty-seven participants ( 30 females and 7 males, age range 19-22 years) took part in this experiment. The data from 1 participant were discarded due to an exceptionally high error rate. The participants were all Cardiff University students and were given course credit for taking part in this study. All had normal or corrected-to-normal vision.

Stimuli. One hundred twenty artificial faces were generated using the InterQuest Faces software package (see Figure 2 for examples). Although initially the faces were randomly generated, a few adjustments were made when the computer produced unacceptable faces - for example, a female-looking face with a mustache. In such cases, the incongruent features were removed. Slight adjustments were made in order to make the faces as different as possible without introducing particularly distinctive features such as scars, glasses, hats, or body piercings. Since InterQuest offers a great number of different facial features (e.g., 1,148 noses, 876 pairs of eyes, 441 hairstyles), it was possible to create a large number of faces that were different in all respects. Female and male faces were approximately equal in number. Also, by using features such as wrinkles, baldness, and hairstyles and by modifying the facial dimensions (e.g., face diameter, interocular distance, heaviness), the perceived ages and races of the artificial faces were varied.

Procedure. The participants were informed that the experiment consisted of three phases: a learning phase, a priming phase, and a posttest phase.

In the learning phase, 30 pairs of faces were presented on a computer screen for $1,500 \mathrm{msec}$ each. The pairs were constructed by randomly assigning 60 faces (out of a set of 120) to these 30 pairs, so that each participant saw a different combination of faces. This was done to keep distinctiveness from affecting learning and subsequent recognition (i.e., some faces are more memorable than others, and therefore are recognized more quickly). The faces of each pair were shown to the participant 12 times, side by side and in a random order. The screen was blank for 1,000 msec between one pair and the next. This process was completely automatic, and no input was required from the participants.

After all 30 pairs of faces had been shown 12 times, the participant was presented with on-screen instructions for the priming phase. In this phase, the face of each pair presented on the left side of the screen (the prime face) was shown in the middle of the screen (centered both horizontally and vertically), where it remained visible for $1,000 \mathrm{msec}$ without any input required from the participant. The purpose of the prime face was not explained; the participant was simply told to pay attention to it without responding. Then, a blank screen with a cross in the middle appeared for $500 \mathrm{msec}$ before the target face was presented. The target face was also presented in the middle of the screen. The participant was instructed to respond only 
to this face as quickly and accurately as possible. In order to report whether or not a face was familiar, the participant had to press the "yes" or "no" key, respectively, marked on the computer keyboard. The computer recorded the response time needed to make this decision.

In the priming phase, there were three conditions: (1) related, in which the two faces of each pair were previously seen paired together (10 trials); (2) unrelated, in which both faces were previously seen paired with other faces (10 trials); and (3) unknown, in which the prime faces had never been seen before but the targets were familiar (10 trials). In these three conditions, the target face was always known, so the participants had to respond to it by pressing the "yes" key. To counterbalance this, an equal number of trials (30) was designed in which the targets were unknown, and therefore the participants had to respond negatively. The conditions of the negative trials were (1) known-unknown, in which prime faces had been seen before but targets were unfamiliar (10 trials); and (2) unknown-unknown, in which both prime and target faces were unfamiliar (20 trials). All 60 trials were presented in a random order. As has already been noted, the faces were randomly and individually assigned to different conditions for each participant. No participant saw the same face assigned more than once to the same condition.

The purpose of the posttest phase was to test whether or not the participants in fact remembered the associate of each face presented in the learning phase. The prime face was presented on the left side of the screen; on the right side, four faces (in a $2 \times 2$ array) were presented, from which the participant had to choose by pressing a corresponding key $(\mathrm{A}, \mathrm{B}, \mathrm{C}$, or $\mathrm{D})$ on the computer keyboard. One face (the target face, which had been paired with the prime face in the learning phase) was the correct answer, but its position in the array was altered from one trial to another. In each array, the remaining three faces consisted of one other known face which in the learning phase had been paired with a face other than the prime, and two unknown faces. The unknown faces had not been presented in the learning phase, but the participants had seen each of them once in the priming phase of the experiment as part of the negative response trials. The familiar (but incorrect) face was always one that had originally been a target face. However, no target face was shown more than once. Since there were 30 known target faces in Experiment 1 and 12 posttest phase trials, each target face occurred only once. However, only 12 prime faces were tested. There was no time limit set for this phase.

\section{Results and Discussion}

The familiarity decision task in the priming phase will be discussed first. All responses above 3,000 msec or below $300 \mathrm{msec}$ (an average of $6.82 \%$ across all participants) were removed from the analyses. Mean response times for correct decisions to the positive faces as a function of type of primes (related vs. unrelated vs. unknown) are shown in Table 1. A one-way repeated measures ANOVA showed a significant effect of prime type $[F(2,35)=7.580, p=$ .001]. Post hoc analysis (Bonferroni test) showed faster responses in the related condition than in the unrelated and unknown conditions. There was no significant difference in latency between unrelated and unknown conditions. The presence of an associatively related prime, where the association was learned through co-occurrence, shortened response time to known faces.

The participants were just briefly exposed to a great number of artificial faces, and, therefore, it is important to present an analysis of their error rates. As for the response times, a one-way repeated measures ANOVA showed a significant effect of prime type $[F(2,35)=6.121, p<$
Table 1

Mean Response Times (in Milliseconds) and Percentages of Error in the Priming Phase for the Positive Conditions of Experiment 1

\begin{tabular}{crrrrr}
\hline & \multicolumn{2}{c}{ Response Time } & & \multicolumn{2}{c}{ Error Rate } \\
\cline { 2 - 3 } \cline { 5 - 6 } Prime Type & \multicolumn{1}{c}{$M$} & $S D$ & & $M$ & $S D$ \\
\hline Related & 963.83 & 311.23 & & 13.51 & 9.77 \\
Unrelated & $1,046.14$ & 395.85 & & 20.18 & 13.41 \\
Unknown & $1,099.08$ & 492.67 & & 22.43 & 15.52 \\
\hline
\end{tabular}

.05]. Post hoc analysis revealed that there were fewer errors in the related than in the unrelated condition, and in the related than in the unknown condition. There was no significant difference between the unrelated and unknown conditions. The presence of an associatively related prime, where the association was learned through co-occurrence, affected both recognition latency and recognition accuracy (see Table 1).

It was hypothesized that co-occurrence is an important factor of association, and the priming effect obtained here supports this hypothesis. The effect on recognition accuracy, however, was not anticipated. Nevertheless, Bruce and Valentine (1986) reported a similar pattern of error distribution across conditions in a priming study conducted with celebrity faces. Thus, since there is evidence in the literature that association facilitates both recognition latency and accuracy, it can be concluded that the effect reported here further supports the claim that cooccurrence is a determining factor of association.

The false alarm rate (i.e., "yes" responses to unknown faces) was $33.9 \%$. The distribution of false alarms between the two negative conditions was relatively equal (18.1\% in the known-unknown condition and $15.8 \%$ unknown-unknown condition).

These results suggest that the human informationprocessing system is able to encode and store a great number of faces after relatively few exposures. Also, the data presented so far indicate that co-occurrence is an important factor in forming associations. The next question is, how strong are the associations produced by co-occurrence? In the posttest phase, the percent of correct responses across all participants was $67.36 \%(S D=17.94)$. When the participants wrongly identified the associate (in the remaining $32.63 \%$ of the post hoc trials), they tended to choose familiar faces $(23.33 \%, S D=14.19)$ over unfamiliar ones $(9.30 \%, S D=6.22)$. These data clearly indicate that the participants showed an above-chance association.

These data, however, do not suggest that the priming effect observed is due to a strong association between the two faces, but rather that there is an episodic memory for the association. It is important to keep in mind that, from the data presented here, it cannot be determined whether the priming effects are produced as a result of an automatic process or as a result of conscious expectancy, since the stimulus onset asynchrony used in this experiment is rather long $(1,500 \mathrm{msec})$. However, this issue is irrelevant to the fact that the associations were learned through cooccurrence. 
Co-occurrence may be a major factor in forming associations between faces, but it is not necessarily the only one. McNeill and Burton (2002) presented strong evidence in support of the idea that associative priming relies critically on shared semantic information. They investigated associative face priming using a semantic decision task (e.g., British or American?) and sex decisions (i.e., male or female?) instead of the familiarity decision task. Although sex decisions did not produce priming, McNeill and Burton reported a priming effect for the semantic decision that was quite similar to that observed for the familiarity decision. Their data clearly argued against an alternative account of semantic priming, such as cooccurrence. Indeed, the semantic decision task used in their experiments does not seem to tap into any perceptual processing. As the authors argued, the face of Paul McCartney might act as a cue in recognizing John Lennon's face as a consequence of their co-occurrence (being seen together often), but if co-occurrence alone produces this effect, why should McCartney's face act as a cue in recognizing Lennon's nationality? Obviously, on the basis of this argument, a perceptual explanation can be easily ruled out.

Thus, several questions are raised: What is the role of semantic relatedness in forming associations? Can semantic relatedness alone produce priming? What is the relationship between co-occurrence and semantic relatedness? In Experiment 2, we explored these issues.

\section{EXPERIMENT 2}

In this experiment, semantic information was introduced alongside the faces in order to investigate the effect of semantic similarity and the interaction between co-occurrence and semantic similarity.

\section{Method}

Participants. Thirty-four Cardiff University students ( 27 females and 7 males, age range 19-22 years) took part in this experiment. They were paid $£ 5$ for their participation. The participants in this experiment had not taken part in the previous one. All the participants had normal or corrected-to-normal vision.

Stimuli. One hundred forty-four artificial faces were used in the experiment. Of these, 120 had been used in the previous experiment, and the remaining 20 were created using the principles described in the previous experiment.

Procedure. The experiment consisted of three phases: a learning phase, a priming phase, and a posttest phase.

In the learning phase, 72 artificial faces (test faces) were presented on the screen along with sets of four semantic dimensions: job, location, sports interest, and music/bands. Each semantic dimension had 38 distinct values (called semantic features; see the Appendix). These different values were randomized and assigned to the semantic dimensions for each face in such a way that no 2 faces shared more than one feature (with the exception of 9 pairs of faces that shared identical sets of semantic features). For example, a face might have been that of a doctor who lives in Dundee, likes rugby, and listens to ABBA. Another face may be that of a lawyer who lives in London, likes soccer, and also listens to ABBA. An example is shown in Figure 3 (see the Appendix for a detailed presentation). The face-semantic feature stimuli were constructed by randomly assigning 72 faces (out of the original set of 144) to these sets of features so that each participant saw a different combination of faces. As in the previous experiment, this was done to prevent distinctiveness from systematically affecting learning and subsequent recognition. The faces and the words appeared on the screen simultaneously.

The 72 faces and their corresponding sets of semantic features (composite face-word stimuli) were presented in pairs on the computer screen. There were 12 blocks. Each pair was presented once per block, and the order of pairs was random. The screen was blank for 1,000 msec between presentations of stimulus pairs. This presentation process was completely automated in that no input from the participants was required. They were instructed to look carefully at the stimuli and to memorize as many of the semantic features associated with each face as possible.

Because the learning phase took almost $1 \mathrm{~h}$ in total, it seemed unlikely that the participants could maintain concentration for the entire length of time and, therefore, a short testing phase occurred at the end of each block (i.e., after each of the 72 faces had been presented), for a total of 12 times. In the short testing phase, a face (randomly selected from the 72 faces displayed in the learning phase) was presented on the left side of the screen and four different sets of semantic features, labeled A, B, C, and D, were presented on the right side of the screen. One set of semantic features belonged to the test face, and the rest of them belonged to other faces presented in the learning phase (i.e., no new, and thus unknown, semantic features were introduced in this phase). The correct answer was shown in a different position each time (Position A, B, C, or D). The face and the semantic features were different for each of the 12 occurrences of the short test phase. The participants had to choose the correct answer within $20 \mathrm{sec}$. The computer recorded the answers and provided the participants with feedback as to whether or not the answer was correct.

When the learning phase was over (roughly $1 \mathrm{~h}$ ), the participants took a 5-min break and were presented with on-screen instructions for the priming phase. In this phase, only faces were presented on the screen, one at a time, without their semantic features. A face (prime) was shown on the screen, where it remained visible for $1,000 \mathrm{msec}$ without any input required from the participants. As in the previous experiment, the purpose of the prime face was not explained to the participants; they were simply told to pay attention to it without responding to it. Then, a fixation cross was presented in the middle of the screen for $500 \mathrm{msec}$ before the target face was presented. The participants were instructed to respond only to this face as quickly and accurately as possible (familiarity decision task). To report whether or not one of the test faces was familiar, the participants had to press the "yes" or "no" key on the computer keyboard. The computer recorded the responses and the times needed to make these responses.

The prime-target pairs occurred in five conditions: (1) related by co-occurrence ( 4 trials), in which the two faces were seen together but shared no semantic features (i.e., their semantic features were different); (2) related by semantic similarity (4 trials), in which both faces were seen previously and shared all semantic features but never co-occurred; (3) related by co-occurrence and semantic similarity ( 4 trials), in which the two faces were seen together and also shared all semantic features; (4) unrelated (12 trials), in which both faces were seen before but never co-occurred and shared no semantic features (instead, they co-occurred and shared all semantic information with another known face); and (5) unknown-known (12 trials), in which the prime faces had never been seen before but the targets were familiar.

In these five conditions ( 36 trials), the target face was always known, so the participants had to respond to it by pressing the "yes" key. To counterbalance this, an equal number of trials (36) were designed in which the target was unknown (and therefore the participants had to respond negatively to them). The conditions of the negative trials were (1) known-unknown, in which prime faces had been seen before but targets were unfamiliar (12 trials); and 


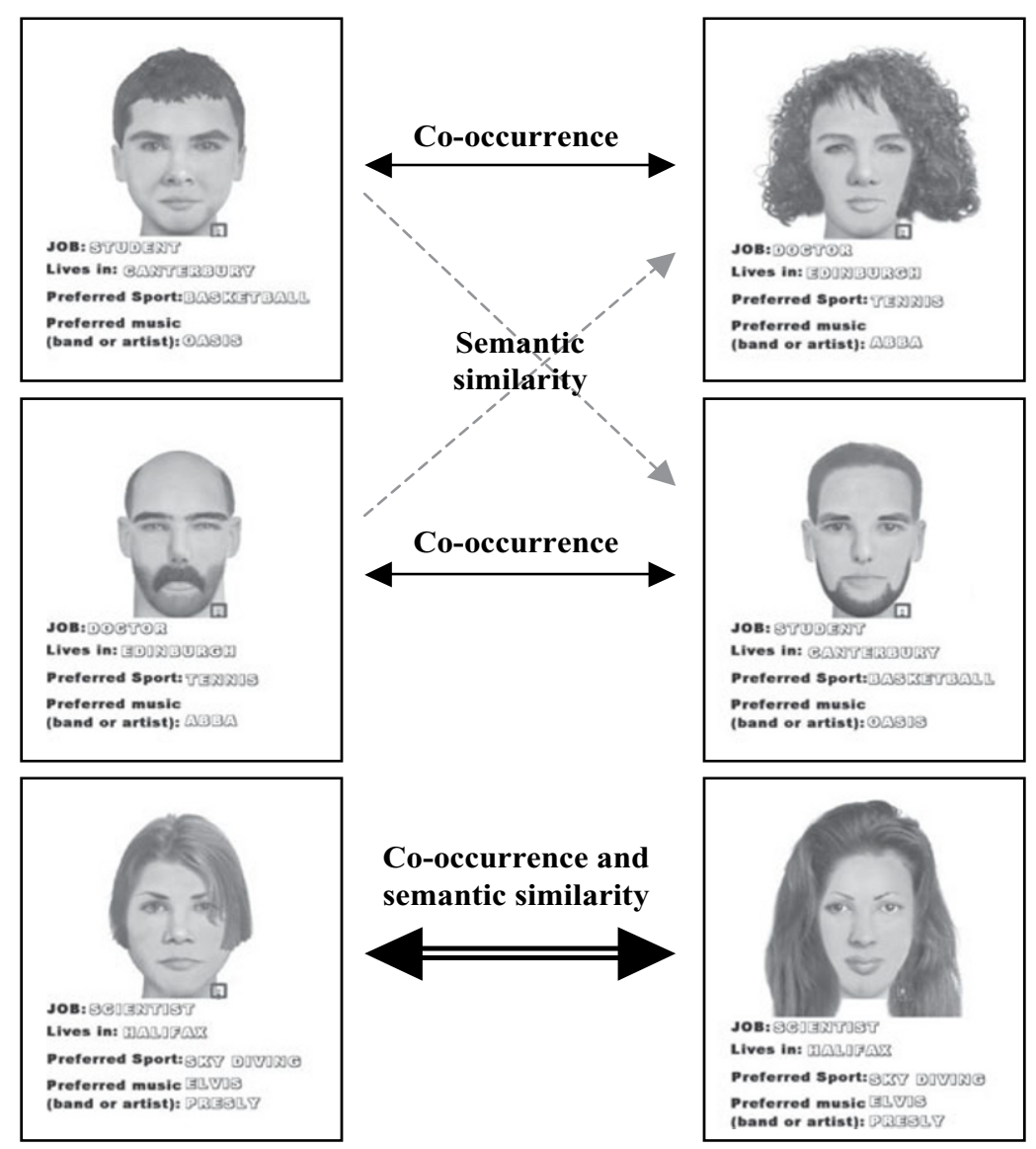

Figure 3. An example of face pairings in Experiment 2.

(2) unknown-unknown, in which both prime and target faces were unfamiliar (24 trials). No semantic features were attached to the unknown faces. The pairs of faces were presented in a random order. As in the previous experiment, the faces were randomly and individually assigned to different conditions for each participant. No participant saw the same face assigned more than once to the same condition.

In the last phase (posttest), the participants were tested in order to see whether, in fact, they remembered the associate of each face presented in the first (learning) phase. As in Experiment 1, the prime face was presented on the left side of the screen, and on the right side four faces (in $2 \times 2$ array) were presented, from which the participants had to choose one. The associations between the prime face and the correct target face were produced by their co-occurrence in the learning phase, by their sharing of all semantic features, or by both co-occurrence and semantic similarity. There were 12 trials in this phase: Four trials tested the strength of the association induced by co-occurrence, 4 tested the strength of the association induced by semantic similarity, and 4 tested the strength of the association induced by both co-occurrence and semantic similarity. In all other respects, Experiment 2 was identical to Experiment 1. Since in Experiment 2 there were 36 known target faces and 12 posttest phase trials, each target face was presented only once. However, only 12 of the prime faces were tested. There was no time limit set for this phase.

\section{Results and Discussion}

All responses above 3,000 msec or below $300 \mathrm{msec}$ (an average of $5.3 \%$ of responses across all participants) were discarded. The correct response means were subjected to a 2 (co-occurrence relationship: related vs. unrelated) $\times$ 2 (semantic relatedness: related vs. unrelated) repeated measures ANOVA. The means are presented in Table 2.

The only significant main effect was that of the cooccurrence relationship variable $[F(1,33)=25.59, p<$ $.001]$. The main effect of the semantic relatedness variable approached significance $[F(1,33)=3.73, p=.06]$. No significant interaction was found between the two variables. The analysis of the simple effects revealed significant effects of co-occurrence for semantically related $[F(1,33)=14.392, p<.01]$ and semantically unrelated $[F(1,33)=11.651, p<.01]$ items. Also, there was a significant effect of semantic relatedness for the co-occurrent

Table 2

Mean Response Times (in Milliseconds) Across Participants for the Correct Responses in the Familiarity Decision Task of Experiment 2

\begin{tabular}{cccccc}
\hline & \multicolumn{3}{c}{ Related by Co-occurrence } \\
\cline { 2 - 3 } \cline { 5 - 6 } Semantic & \multicolumn{2}{c}{ Yes } & & \multicolumn{2}{c}{ No } \\
\cline { 2 - 3 } \cline { 5 - 6 } Relatedness & $M$ & $S D$ & & $M$ & $S D$ \\
\hline Related & 704.19 & 104.44 & & 775.11 & 127.25 \\
Unrelated & 729.55 & 194.29 & & 826.12 & 163.38 \\
\hline
\end{tabular}


Table 3

Mean Percentages of Error Across Participants in the Priming Phase of Experiment 2 Related by Co-occurrence

\begin{tabular}{ccrrrr} 
& \multicolumn{3}{c}{ Related by Co-occurrence } \\
\cline { 2 - 3 } \cline { 5 - 6 } Semantic & \multicolumn{2}{c}{ Yes } & & \multicolumn{2}{c}{ No } \\
\cline { 2 - 3 } \cline { 5 - 6 } Relatedness & $M$ & \multicolumn{1}{c}{$S D$} & & \multicolumn{1}{c}{$S$} & \multicolumn{1}{c}{$S D$} \\
\hline Related & 2.94 & 8.17 & & 10.26 \\
Unrelated & 8.09 & 11.87 & & 21.57 & 15.36 \\
\hline
\end{tabular}

items $[F(1,33)=6.324, p<.05]$ but not for the non-cooccurrent items $[F(1,33)<1]$.

The data presented here suggest again that co-occurrence plays an important role in associative priming, which parallels its role in semantic relatedness.

The next question is whether or not the addition of semantic information contributes to greater accuracy in face recognition. As can be observed in Table 3, the error rate decreased significantly in the semantically related conditions (i.e., related by semantic similarity, and related by both semantic similarity and co-occurrence). A $2 \times 2$ repeated measures ANOVA was computed as previously. The main effect of the co-occurrence relationship variable was significant $[F(1,33)=61.49, p<.001]$, as was the main effect of semantic relatedness $[F(1,33)=58.73$, $p<.001]$. The interaction between the two variables was also significant $[F(1,33)=73.94, p<.001]$. The analysis of the simple effects revealed significant effects of cooccurrence for the semantically related $[F(1,33)=55.006$, $p<.001]$ and semantically unrelated $[F(1,33)=56.842$, $p<.001]$ items. Also, there was a significant effect of semantic relatedness for the co-occurrent items $[F(1,33)=$ $4.9775, p<.05]$ but not for the non-co-occurrent items $[F(1,33)=1.585, p>.05]$.

The false alarm rate was $28.3 \%(16.3 \%$ in the knownunknown condition and $12 \%$ unknown-unknown condition). The error rates for the posttest phase were high but comparable to those found in the previous experiment. The mean error rates across participants are presented in Table 4. The posttest data showed that the participants made the majority of errors by choosing familiar over unfamiliar faces, indicating a pattern similar to that of Experiment 1 . These data clearly indicate that the participants showed an above-chance association. The error rate seems to decrease with the addition of semantic relatedness, which suggests that semantic information plays a crucial role in the correct retrieval of associates.

As we originally hypothesized, although the initial association between two faces can be built solely on co- occurrence (as was shown in Experiment 1), semantic information seems to strengthen the association. A potential issue in this experiment, however, is the strength of the associations formed in this artificial way (i.e., by presenting faces together with a set of semantic features). Because $85 \%$ of our participants were able to memorize the set of semantic features associated with the faces with a reasonable level of accuracy $(75 \%-100 \%)$, it is clear that the task is manageable and the participants performed satisfactorily. However, it can be argued that the strength of the association between the face and the semantic features may have been quite low, for two main reasons. The first is the artificiality of the stimuli. For example, a computer-generated character cannot really be a carpenter in Cardiff who likes rugby and listens to Madonna. A real character's semantic attributes are learned quite differently. For example, one knows that David Beckham is a soccer player not from seeing Beckham's picture in the newspaper together with the word soccer, but because one has seen Beckham play soccer. Thus, the association between Beckham's face and his job is quite strong and reinforced in many hours of watching soccer. This brings us to the second reason for the weak association between the faces and their semantic features - namely, the form of presentation of the semantic information. It is well known that humans prefer a narrative approach over an expository one (Wilkes, 1997). One forms a "semantic database" about David Beckham by reading stories of his success or his private life, or comments about his performance on the field. In our experiment, the semantic information was presented without a context or story line and in a very condensed style, which probably led to a poorer linkage between it and the associated faces.

\section{GENERAL DISCUSSION}

The first experiment of the present study showed that an associative priming effect, similar in magnitude to the effects reported in the literature (e.g., Barry et al., 1998; Bruce \& Valentine, 1986; Young et al., 1994), can be obtained solely by the co-occurrence of computer-generated faces, which have no semantic background that could explain their association. It is likely that association is built gradually, through an associative learning process, within the phase of acquisition of new faces. The novelty of this study consists in its role in investigating the processes that occur together with face acquisition or that emerge as a consequence of the conditions in which face acquisition took place, and in its independence of the recognition of

Table 4

Mean Percentages of Error in the Posttest Phase of Experiment 2

\begin{tabular}{lccccc}
\hline \multirow{2}{*}{\multicolumn{1}{c}{ Condition }} & \multicolumn{2}{c}{ Total Error Rate } & & \multicolumn{2}{c}{ Type of Error (\%) } \\
\cline { 2 - 3 } \cline { 5 - 6 } \cline { 5 - 6 } & $M$ & $S D$ & & Familiar & Unfamiliar \\
\hline Related by co-occurrence & 30.7 & 17.2 & & 23 & 7.7 \\
Semantically related & 22.5 & 14.22 & & 12.4 & 10.1 \\
Related by both & 18.5 & 12.15 & & 10 & 8.5 \\
\hline
\end{tabular}


faces that are already known. In the psychological literature in general, the fact that repeated exposure to stimuli eventually contributes to more accurate and faster recognition of those stimuli is quite widely accepted. However, at least in the face recognition literature, it is not entirely clear to what degree this exposure could lead to association. Also, the commonly accepted view is that associations are formed by sharing background information, be it purely semantic (see, e.g., Bruce \& Valentine, 1986; Cabeza, Burton, Kelly, \& Akamatsu, 1997; Carson \& Burton, 2001; McNeill \& Burton, 2002; Schweinberger, 1995; Young et al., 1994) or biographical (Barry et al., 1998). The experiments presented in this article demonstrate that associations can be formed solely on the basis of co-occurrence. However, this does not mean that the role of semantic relatedness can be readily dismissed. As was hypothesized in the introduction, semantic and associative priming are two distinct forms of priming. In Experiment 2, a priming effect based on semantic relatedness was observed even though the respective faces never co-occurred. This finding further supports a multitude of studies suggesting that associations can be formed between faces sharing semantic features. However, as can be seen in Table 2, the priming effect based solely on semantic relatedness is weaker than that produced by cooccurrence. Similar results have been reported in the word literature by Lupker (1984) and, more recently, by Alario et al. (2000).

We must acknowledge as a limitation of this study the fact that only one third of the negative trials were in the known-unknown condition; known targets were preceded by known primes in two thirds of the trials. This may introduce a bias in the participants' responses, since prime familiarity could provide some information about the probability of target familiarity. Thus, the participants might have shown longer response times to the unknown targets. Although we acknowledge this design flaw, we note that it does not present a fatal problem for the experiment as a whole because it does not affect the comparisons between the positive conditions.

The issue of what constitutes real semantic information is quite complicated. As a general conclusion, it seems quite clear that some information is more important than other information and some strands are interrelated and strongly linked whereas others are weakly linked. The hierarchies probably differ from one individual to another. Therefore, it is difficult to determine which bit of information is playing a major role in forming associative links. Co-occurrence, however, has a straightforward effect. The more two faces co-occur, the stronger will be the association between them.

Another important issue is that the results presented here cannot be fitted in the IAC model of face recognition or in either of the two main IAC-type models published to date: IAC-L (Burton, 1994) and IACAPA (Stevenage \& Lewis, 2002). The core assumption of the IAC model is that semantic relatedness alone (encoded by several SIUs) is the cause of the associative priming effect. However, Experi- ment 1 showed that priming can be obtained without semantic information. One possible solution is to incorporate separate nodes designed to encode episodic events, such as co-occurrence. Consequently, two PINs could be linked to several SIUs and one episodic node. Such a model could explain the data from both experiments described herenamely, priming effects based on (1) co-occurrence only and (2) co-occurrence and semantic relatedness. However, such a model would not explain how co-occurrence influences the process of face acquisition and the formation of associations between faces that co-occurred.

The data presented thus far do not support the idea that the locus of priming based solely on co-occurrence is at either the PIN level or the FRU level. Further experiments (in progress) that use computer-generated faces and names to produce cross-modal priming could settle this argument. Furthermore, the data presented here pose another major difficulty for any model of face acquisition and processing: the need to explain how semantic information is acquired.

To conclude, the data we have presented here support the idea that semantic and associative priming are two different phenomena. Co-occurrence seems to be the determining factor of associative priming, whereas semantic similarity plays the essential role in semantic priming. Unfortunately, the manipulations in the experiments presented here lacked the power to sustain the claim of additivity between co-occurrence and semantic relatedness. However, we would like to propose that in some priming studies based on known faces presented in the literature, co-occurrence and semantic information were intrinsically linked and both factors contributed to the formation of associations. It is possible that in some cases co-occurrence strengthens the links between bits of semantic information (e.g., partnerships) and may even help in the formation of semantic categories.

\section{REFERENCES}

Alario, F. X., Segui, J., \& Ferrand, L. (2000). Semantic and associative priming in picture naming. Quarterly Journal of Experimental Psychology, 53A, 741-764.

Barry, C., Johnston, R. A., \& Scanlan, L. C. (1998). Are faces "special" objects? Associative and semantic priming of face and object recognition and naming. Quarterly Journal of Experimental Psychology, 51A, 853-882.

Bruce, V., \& VAlentine, T. (1986). Semantic priming of familiar faces. Quarterly Journal of Experimental Psychology, 38A, 125-150.

Burton, A. M. (1994). Learning new faces in an interactive activation and competition model. Visual Cognition, 1, 313-348.

Burton, A. M., Bruce, V., \& Johnston R. A. (1990). Understanding face recognition with an interactive activation model. British Journal of Psychology, 81, 361-380.

Cabeza, R., Burton, A. M., Kelly, S. W., \& Akamatsu, S. (1997). Investigating the relation between imagery and perception: Evidence from face priming. Quarterly Journal of Experimental Psychology, 50A, 274-289.

Carson, D. R., \& Burton, A. M. (2001). Semantic priming of person recognition: Categorial priming may be a weaker form of the associative priming effect. Quarterly Journal of Experimental Psychology, 54A, 1155-1179.

Collins, A. M., \& Loftus, E. F. (1975). A spreading activation theory of semantic processing. Psychological Review, 82, 407-428. 
HaY, D. C., \& Young A. W. (1982). The human face. In A. W. Ellis (Ed.), Normality and pathology in cognitive functions (pp. 173-202). London: Academic Press.

Hutchison, K. A. (2003). Is semantic priming due to association strength or feature overlap? A microanalytic review. Psychonomic Bulletin \& Review, 10, 785-813.

Kroll, J. F., \& PotTer, M. C. (1984). Recognizing words, pictures and concepts: A comparison of lexical, object and reality decisions. Journal of Verbal Learning \& Verbal Behavior, 23, 39-66.

LuCAS, M. (2000). Semantic priming without association: A meta-analytic review. Psychonomic Bulletin \& Review, 7, 618-630.

LUPKER, S. J. (1984). Semantic priming without association: A second look. Journal of Verbal Learning \& Verbal Behavior, 23, 709-733.

McNeILl, A., \& BURTON A. M. (2002). The locus of semantic priming effects in person recognition. Quarterly Journal of Experimental Psychology, 55A, 1141-1156.

Moss, H. E., Ostrin, R. K., Tyler, L. K., \& Marslen-Wilson, W. D. (1995). Accessing different types of lexical semantic information:
Evidence from priming. Journal of Experimental Psychology: Learning, Memory, \& Cognition, 21, 863-883.

SCHWEINBERGER, S. R. (1995). Personal name recognition and associative priming in patients with unilateral brain damage. Brain \& Cognition, 28, 23-35.

SCHWEINBERGER, S. R. (1996). How Gorbachev primed Yeltsin: Analyses of associative priming in person recognition by means of reaction times and event-related brain potentials. Journal of Experimental Psychology: Learning, Memory, \& Cognition, 22, 1383-1407.

Stevenage, S. V., \& LewIS, H. L. (2002). Understanding person acquisition using an interactive activation and competition network. Visual Cognition, 9, 839-867.

WiLKes, A. L. (1997). Management of text comprehension. In A. L. Wilkes (Ed.), Knowledge in minds: Individual and collective processes in cognition (pp. 237-263). Hove, U.K.: Psychology Press.

Young, A. W., Flude, B. M., Hellawell, D. J., \& Ellis, A. W. (1994). The nature of semantic priming effects in the recognition of familiar people. British Journal of Psychology, 85, 393-411.

\section{APPENDIX \\ Design of Experiment 2}

\section{Learning Phase}

Seventy-two faces (represented in Table A1 by the numbers 1-72) and their semantic features (represented in Table A1 by one or two letters or by letter/number combinations) were used in the learning phase. The faces used in the experiment were randomly assigned to the positions represented by the numbers $1-72$.

Table A1

Face-Feature Sets Used in Experiment 2

\begin{tabular}{llllll}
\hline $1 \mathrm{~A}-2 \mathrm{~B}$ & $13 \mathrm{H}-14 \mathrm{G}$ & $25 \mathrm{~N}-26 \mathrm{O}$ & $37 \mathrm{Z}-38 \mathrm{~N} 2$ & $49 \mathrm{Y} 2-50 \mathrm{2}$ & $61 \mathrm{KK}-62 \mathrm{LL}$ \\
$3 \mathrm{C}-4 \mathrm{~A}$ & $15 \mathrm{I}-16 \mathrm{~F}$ & $27 \mathrm{P}-28 \mathrm{Q}$ & $39 \mathrm{O} 2-40 \mathrm{P} 2$ & $51 \mathrm{AA}-52 \mathrm{BB}$ & $63 \mathrm{AB}-64 \mathrm{CD}$ \\
$5 \mathrm{D}-6 \mathrm{C}$ & $17 \mathrm{~J}-18 \mathrm{~J}$ & $29 \mathrm{R}-30 \mathrm{~S}$ & $41 \mathrm{Q} 2-42 \mathrm{R} 2$ & $53 \mathrm{CC}-54 \mathrm{DD}$ & $65 \mathrm{EF}-66 \mathrm{GH}$ \\
$7 \mathrm{E}-8 \mathrm{D}$ & $19 \mathrm{~K}-20 \mathrm{~K}$ & $31 \mathrm{~T}-32 \mathrm{U}$ & $43 \mathrm{~S} 2-44 \mathrm{~T} 2$ & $55 \mathrm{EE}-56 \mathrm{FF}$ & $67 \mathrm{IJ}-68 \mathrm{KL}$ \\
$9 \mathrm{~F}-10 \mathrm{I}$ & $21 \mathrm{~L}-22 \mathrm{~L}$ & $33 \mathrm{~V}-34 \mathrm{~W}$ & $45 \mathrm{U}-46 \mathrm{~V} 2$ & $57 \mathrm{GG}-58 \mathrm{HH}$ & $69 \mathrm{MN}-70 \mathrm{OP}$ \\
$11 \mathrm{G}-12 \mathrm{H}$ & $23 \mathrm{M}-24 \mathrm{M}$ & $35 \mathrm{X}-36 \mathrm{Y}$ & $47 \mathrm{~W}-48 \mathrm{X} 2$ & $59 \mathrm{II}-60 J \mathrm{~J}$ & $71 \mathrm{QR}-72 \mathrm{ST}$ \\
\hline
\end{tabular}

Semantic sets A-Z and AA-LL ( 38 sets) are completely different. Thus, there are 38 different jobs, 38 different locations, 38 different sports interests, and 38 different music/band preferences. There are also 13 semantic sets (N2 to Z2, labeled with letter/number combinations) and 10 sets (AB to ST, labeled with two letters) that were constructed by randomly assigning features from Sets A-KK in such a way that no two sets share more than one feature.

One question that might be asked is why we did not create 61 different jobs, 61 different locations, 61 different sport interests, and 61 different music preferences. In pilot work, we found out that participants cannot learn so many different features, especially in an artificial task lacking an underlying story and other features of real-life situations. In real-life situations, people learn semantic features about other people not as separate labels but in a context, and they use contextual or social cues to link these features in a more or less coherent story. Since contextual or social cues are absent in our experiment, it is very likely that participants cannot use a mnemonic strategy to learn such a great amount of information effectively. Second, it was hard to find 61 different sports that all of our participants knew, 61 music bands that are equally famous, and so forth. Thus, we had to somehow reduce the number of values (features) for each semantic dimension without compromising the semantic unrelatedness between the faces. The fact that two semantically unrelated faces share a semantic feature is not enough to make them associates. In any case, if two faces shared a semantic feature, they were never put into the same prime-target pair in either the learning phase or the priming phase. 
APPENDIX (Continued)

Priming Phase

The 72 faces used in the learning phase and an additional 72 unknown faces were used in the priming phase of Experiment 2. The faces were paired as shown in Tables A2 and A3.

Table A2

Face Pairs in Positive Trials (on Which Correct Response Is Always "Yes")

\begin{tabular}{cccc}
\hline \multicolumn{1}{c}{ Condition } & Related & Unrelated & Unknown-Known \\
\hline Co-occurrence & $1-2$ & $25-48$ & $85-50$ \\
& $3-4$ & $27-46$ & $86-52$ \\
& $5-6$ & $29-44$ & $87-54$ \\
Semantic similarity & $7-8$ & $31-42$ & $88-56$ \\
& $9-16$ & $33-40$ & $89-58$ \\
& $11-14$ & $35-38$ & $90-60$ \\
Both & $13-12$ & $37-36$ & $91-62$ \\
& $15-10$ & $39-34$ & $92-64$ \\
& $17-18$ & $41-32$ & $93-66$ \\
& $19-20$ & $43-30$ & $94-68$ \\
& $21-22$ & $45-28$ & $95-70$ \\
& $23-24$ & $47-26$ & $96-72$ \\
\hline
\end{tabular}

Table A3

Face Pairs in Negative Trials (on Which Correct Response Is Always "No")

Known-Unknown Unknown-Unknown

\begin{tabular}{lcc}
$49-73$ & $97-98$ & $121-122$ \\
$51-74$ & $99-100$ & $123-124$ \\
$53-75$ & $101-102$ & $125-126$ \\
$55-76$ & $103-104$ & $127-128$ \\
$57-77$ & $105-106$ & $129-130$ \\
$59-78$ & $107-108$ & $131-132$ \\
$61-79$ & $109-110$ & $133-134$ \\
$63-80$ & $111-112$ & $135-136$ \\
$65-81$ & $113-114$ & $137-138$ \\
$67-82$ & $115-116$ & $139-140$ \\
$69-83$ & $117-118$ & $141-142$ \\
$71-84$ & $119-120$ & $143-144$ \\
\hline
\end{tabular}

(Manuscript received August 11, 2003; revision accepted for publication June 28, 2005.) 